\title{
Collaboration between Network Players of Information Centric Network: An Engineering-Economic Analysis
}

\author{
M. Arifuzzaman*, K. Yu and T. Sato** \\ Graduate School of Global Information and Telecommunication Studies, \\ Waseda University, Tokyo, Japan \\ E-mail: \{arif; yukeping\}@fuji.waseda.jp; t-sato@waseda.jp \\ *Student Member, IEEE; **Fellow, IEEE
}

Received: 14 October 2014;

Accepted: 9 March 2015

\begin{abstract}
Internet use is dominated by content distribution and retrieval. As there is a rapid explosion of web-based content, it becomes challenging to provide quality of experience to the end-user. To efficiently handle the utmost growth of the Internet content, in business perspective, there is a clear increase in Content Delivery Networking (CDN). On the other hand, in research perspective it is the Information Centric Networking (ICN) which emerged as an alternative to the current host-to-host communication paradigm and has the potentials of distribution and retrieval of contents more efficiently. This article is an initiative to find some rooms where the ICN and the CDN can meet together. In ICN, a potential strategy by the publisher ignores CDN providers and has direct connections with ISPs. However we believe that, at the stage of incremental deployment of ICN, there is no scope of ignoring CDN. Besides, it is true that it will take time to deploy ICN in Internet scale. So, for achieving big economy of scale publisher will choose CDN as a means for its content distribution. In this paper we present two possible content distribution models for ICN. We formulate game theoretic model for the network players for ICN architecture based on our proposed content distribution model. Firstly, we
\end{abstract}

Journal of ICT, Vol. 2, 201-222.

doi: $10.13052 /$ jicts2245-800X.231

(C) 2015 River Publishers. All rights reserved. 
present two player game model between Publisher and Telco CDN. And we discuss the way of Revenue sharing between Telco CDN and Telco CDN (or Telcos or ISPs); where we assume that interconnection among Telco CDNs is possible. Following, we enhance the game among three major network players (Publisher, Global CDN and Telco CDN) of ICN to analyze the way of the economic incentives sharing among them. We also present a solution for Live Streaming Media broadcast in ICN and analyze the economic part with a decision tree. Besides, we identify some standardization issue in ICN architecture and we emphasize on the need for a common standard for content routers $(\mathrm{CR})$ so that as a node in the ICN, CR ensure scalable content delivery as well as its functionalities match with the Internet open standard philosophy.

Keywords: Information Centric Networking (ICN), Content Delivery Network (CDN), Telco CDN, Telco (Telecommunications Operator), Internet Service Provider (ISP), Quality of Experience (QoE).

\section{Introduction}

The internet is developing into content network i,e, a video and media network. CDNs play a significant role in the current Internet to optimize the delivery of content. Truly speaking without CDN the current internet would have died already due to lack of capacity. In most of the cases the large content providers use to pay CDNs to deliver their content more efficiently and with guaranteed latencies. ISPs collaborate with CDNs in order to perform such optimized delivery [1]. CDNs proactively push content to servers and then manipulate the Domain Name System (DNS) so as to serve users from nearby servers [2].

On the other hand, Content Centric Network (CCN) [3] is a new paradigm which aims to replace machines by content in the networking communication model. In CCN architecture the network layer provides with content besides providing merely communication channel between hosts. In the PSIRP [4], content-centric networking is used as a base for defining a new architecture. With the Publish-Subscribe internet (PSI) architecture, Information Centric Networking (ICN) focuses on content rather than end point communication. In PSI, any requested information item can be served from the nearby cache or other possible source including replication points. By the nature of the design principle of ICN we can easily recognize that the benefits of ICN dependent on the extensive cache structure in the network. However, it is 
true that without some explicit monetary gain the network players will not be interested to provide extensive cache structure, neither will it be interested to accommodate a totally new internet architecture. Rather the network player will try to be steadfast with the current internet architecture and continue trying to bridge the gap between the host centric model and the future internet needs by patching to the internet architecture including Mobile IP, CDNs, P2P overlays etc. In this article, we clarify some economic issue of Information centric networking. We recognize the current $\mathrm{CDN}$ market scenario to make an engineering economic analysis of ICN. We believe our paper is a good starting point to think the business relationship among different existing network players in the ICN architecture. The major network players we consider are Publisher, Global CDN (example Akamai, Limelight Networks, CDNetworks etc), Telco CDN (CDN developed in an access operator's network i.e., by telecom operators or ISP) and Telcos (or ISPs). The rest of the paper is organized as follows: In Section 2, some related works are discussed. In Section 3, we focus on the standardization issue of ICN architecture. We describe our proposed Content Distribution model for ICN in Section 4. In Section 5, gives the game theoretic model between two player; Publisher and Telco CDN. In Section 6, we enhance the game model between three player; Publisher, Global CDN and Telco CDN and show necessary analysis. In Section 7, we present a solution for Live Streaming Media broadcast in ICN and analyze the economic part with a decision tree. Finally, Section 8 concludes the paper with a summary and some scope of future work.

\section{Related Work}

Information-Centric Networking (ICN) has gained substantial attention to the research community in recent years as candidate architecture for the future internet. There have been some efforts to address the economic and business aspect of the information centric networking. In [5], the significance of the socio-economic issues in evaluating the future Internet design is clarified and vividly explained. The way of resolving conflicts between the various constellations of stakeholder interests, conflicts etc. are also analyzed in ICN perspective. In [6], the authors present simple economic model to assess the incentives of various network players to establish distributed storage architecture to realize the Information Centric Network. Economic incentives for network players in deploying ICN-based architecture are focused in [7]. Authors also presented a qualitative analysis very precisely. A hierarchical cooperative game model for Resource and revenue sharing 
among cloud providers has been developed in [8]. Authors have shown that the cooperation and coalition formation can lead to higher profit for the cloud providers. Several technical and business aspects of Content Delivery Network are analyzed from a game theoretic perspective in [9]. For the booming internet-based video consumption, the new revenue opportunity for the Telecommunication and cable providers is unveiled in [10]. In [11], the authors survey the ecosystem for each of the three technical solutions that are proposed in three work packages i.e. Network of Information (NetInf), Open Connectivity Services (OConS) and Cloud Networking (CloNe) of the SAIL (Scalable and Adaptable Internet Solutions) project. Besides the technical architecture, the business architecture of the SAIL project is also precisely presented. In [12], the caching problem in P2P systems is modeled by game theory and authors have shown that the game can reach a Nash Equilibrium. In [13], by using a series of novel payment mechanism, a formal game theoretic model for P2P network is constructed by the authors. The design implications of the case where all users act selfishly to maximize their personal gain are investigated. The paper also analyzed equilibrium of strategies taken by different players. In [14], an analysis of the decision making problem of caching contents by the network players of Information Centric Networking (ICN) is shown. A solution for Live Streaming Media broadcast in ICN and analysis of the economic part with a decision tree is also presented. Besides, the paper also discusses some standardization issue in ICN architecture.

\section{Standardization Issue of ICN Architecture}

Though the ICN is its infancy, it is candidate architecture for the future internet. Currently, numerous projects are going on under the ICN theme. They vary in their design aspects. We cite here few differences between two major Information Centric Networking project CCN [3] and PSIRP [4] as an example. In case of naming the content, $\mathrm{CCN}$ uses hierarchical naming and PSIRP uses flat naming. For security, CCN needs to trust signing key to establish integrity, where in case of PSIRP it is self- certifying. For name resolution and routing, name based routing using longest prefix of hierarchical names is used in CCN. On the other hand, a rendezvous function is used, within a specified scope to solve the issue in PIRSP. For transport and caching, CCN transport using named based routing; finds cached object through local search as well as on the path to the publisher. For PIRSP, transport routing and forwarding use separate forwarding identifier. So, the two projects vary in 
their design concepts. Similar variations are also found in other projects like DONA [15], NetInf [16] etc.

Though at some points like naming, security, etc deserve early initiative of standardization, the interest of this article on standardization issue is on the major network player interoperability perspective. Now the question is at which point or level of a network (or operator) we expect to communicate with other network (or operator) in ICN architecture. Before explain that, we recall the brief scenario of the extensive cache structure of ICN. It is evident from the ICN literature that to achieve the optimum benefit from the ICN, all major network players have to establish cache in the strategic locations of their network.

Five types of network players, i, e., Internet Service Provider, ISP (Tier 3 ISPs or Tier 2 ISPs), Transit Network (Tier 1 and Tier 2 ISPs), Content Distribution Networks (CDNs) and Large Scale Savvy content provider and publisher can be considered as major network players in ICN. Among them all network players will install cache other that publishers. How cache deployment can bring benefits to the different network players are explained precisely in paper [4]. Now, we know, in ICN architecture, the content will be retrieved from the nearby content router, CR (cache) whether the CR belongs to the same operator, or not. However, the proprietary nature of most content routing designs in use today makes them undesirable for global use and is in conflict with the Internet open standard philosophy.

Since to achieve the optimum benefit from the ICN design, edge router (CR) compatibility and the interconnection between the CR is very important, we believe, an early initiative should be taken by the standardization body to find the standard for CR. On an abstract level, Information centric networking can be compared to a network with CDN server everywhere. So, the lessons from CDNs can rightly explain the necessity of the standardization of the router to router communication in ICN. As we know CDN interconnection can provide benefits to $\mathrm{CDN}$ service providers as well as to end users. Since its legacy, CDNs were realized using proprietary technology, open interface support for connecting with other CDNs has been ignored. Even though several CDNs could be interconnected, it is still challenging to achieve interconnection at operational level such as exchange billing information and so on. CDNi-WG is pursuing solutions to these problems.

To sum we can say that an early standardization initiative on economic and technical phenomenon as well as a policy initiative can significantly contribute in the maturation process of the ICN as future internet architecture. 


\section{Proposed Content Distribution Model for ICN and Related Conceptual Overview}

In this section, we propose rational content distribution model which suit with the Information Centric Network. We use this proposed content distribution model for modeling the game among the network players. In current internet architecture, typically client-server model or Global CDN or combination of both Global CDN and client-server model are used by the majority of the content providers or publishers. In case of clientserver model the publisher use their own or leased servers for their content distribution. The key considerations of the content provider's current content distribution system are cost effectiveness, reliability, scalability, flexibility and fast content distribution. Generally, publisher choose client server model because of the cost effectiveness of the model. Nevertheless, publishers choose Global CDN in the case where large traffic amounts are expected. Besides, in case of live streaming publishers choose Global CDN. We assume that for the ICN architecture, publisher will consider the same parameters (cost efficiency, reliability, scalability etc.) for selecting a contentment distribution model. Like current trend, if the publisher gains more users, for the scalability problem publisher will be interested to buy services from Global CDNs. Besides, in ICN, local ISPs will be equipped with in network cache; hence the response time (user perceived time between sending a request and receiving the response) will be minimized resulting Quality of Experience (QoE) for the users. So, compared to the current internet architecture where cost effectiveness is given the highest priority in choosing a content distribution model by the publisher, in ICN faster content distribution will be equally important.

We propose two possible options for publisher to disseminate its content as follows: In the first notion we assume that the publisher can ignore Global CDN providers and have direct connections with Telco CDN. Telco CDN will ensure the big economy of scale for the publisher by Telco CDN interconnection/Federation based on 'Smart Pipe Concept' [17]. In the second notion we assume that publisher will choose Global CDN for its content disseminations and for big economy of scale. And Telco CDN, Telcos and ISPs will ensure last mile connectivity and optimized delivery of the content where they will receive revenue from Global CDN for providing Quality of Experience (QoE). The scenario is briefly depicted in Figure 1.

Now, in ICN the ISP (or Telco) will serve the content to the client possibly in following ways. Each time after receiving a request from the client, the content is fetched from the source server or the Telco's CDN 


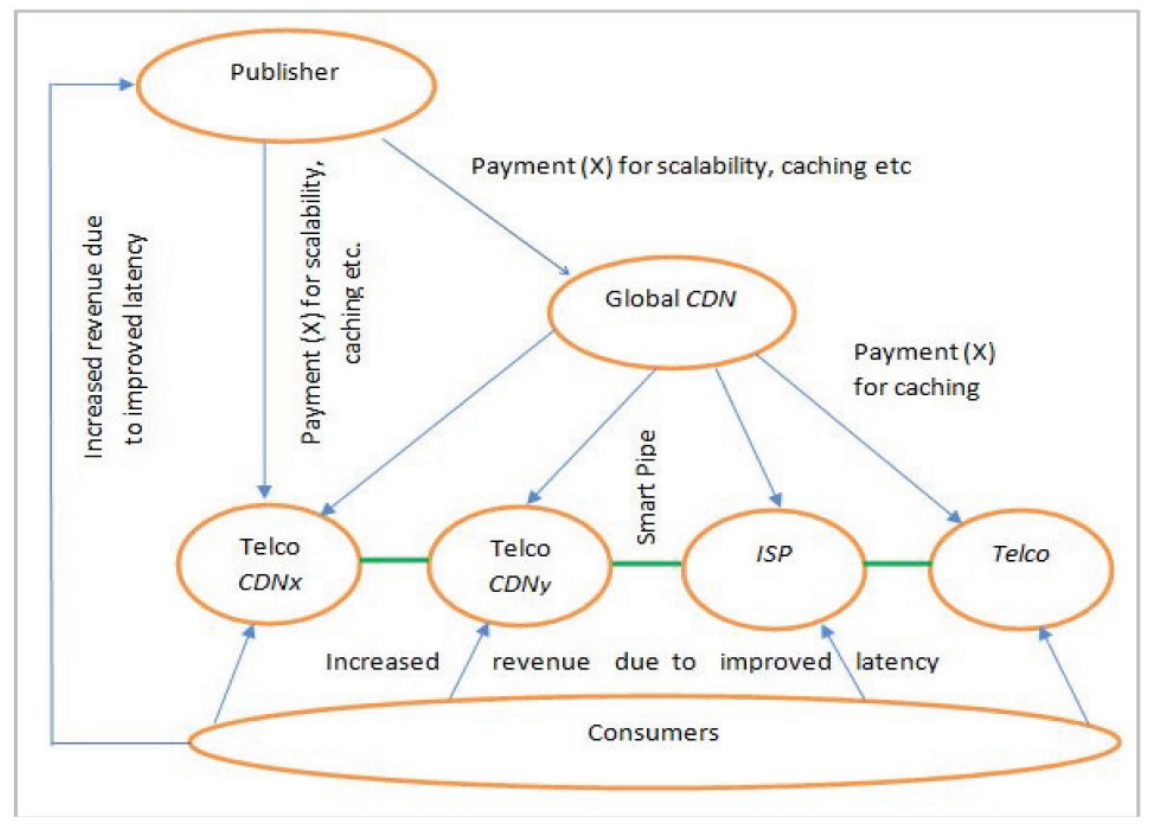

Figure 1 Possible content distribution scenario in ICN architecture

server (its own or other's) or Global CDN's server and serves the client. The other option is to keep the copy (either on-path-caching or off-path caching) of the content in the Content router (of ISP's local network) and serve the client with this cached content for all subsequent requests. Now, typically publisher's content will be managed, cached and hence served by Telcos or ISPs with an objective to minimize the total transit traffic of the Telcos or ISPs. The conventional caching algorithm is that, for the incoming traffic gradually replaces the least popular contents by the contents that are becoming popular. The content popularity typically measured based on its request frequency. Though this strategy is equally valid for ICN, however in addition to that, even though the content is not popular enough, the Telcos or ISPs can provide caching service for the content of selective publishers (due to agreement or good business relations through $\mathrm{CDN}$ ) by deriving propriety algorithms. The examples of other type of contents (information) like warning of disasters; vaccination information etc. deserve priority treatment (providing on-path/off-path caching service) by the Telcos \& ISPs as an obligation of social responsibility. 
Figure 1, shows the relationship between different network players in a content distribution model of ICN where publisher choose Global CDN or Telco CDN for its content distribution. Though, the Telcos/ISPs delivery the content to the end user, the publisher cannot pay directly to Telcos or ISPs. It will increase the transaction cost of publisher since in that case publisher has to make a business relation with several/many Telcos and ISPs. So, a rational assumption is that publisher will pay to the Global CDN or Telco CDN in order to publish and delivery of its content and caching. By caching more popular content the Telcos and ISPs can maximize its cash inflow from the customer side. On the other hand, by caching the CDN's (Global or Telco) replicated content Telcos and ISPs can maximize its cash inflow from the CDN side. It is realistic that, content with the similar popularity Telcos and ISPs will be interested to cache a content which can minimize more transit traffic i.e., if the content need to be fetched from the remote server, it will be cached rather the content that will be served by the CDN cache server at the proximity. With this strategy Telcos and ISPs can avoid higher delivery time for content as well. In the end, Telcos and ISPs will face the optimization problem to maximize the revenue to support its client by providing caching service for the popular content and to providing caching service to the chosen publisher's content which is not popular yet but publisher paid for the caching service through Global CDN or Telco CDN.

Now for the ISPs, if they are not intent to institute the caching structure, they must invite the third party to establish cache structure inside their network. Without caching services they cannot provide enhance user experience to their clients hence cannot keep pace with the services of other service providers. Since CDN (Global or Telco) are already doing the alike and they have expertise, they can establish cache functionalities for the small ISPs and maximize their cash inflow.

\section{Two Player Game Model for ICN}

In this section, we formulate a game theoretic model for the network players for ICN architecture. Firstly we propose a game theoretical model for the two network players; Publisher and Telco CDN. Secondly, we briefly discuss the revenue sharing scenario between the Telco CDN (who has agreement with the publisher for content dissemination) and other peering Telco CDNs (or Telcos or ISPs).

The game is based on the proposed content distribution model that we described in Section 3. The whole game is actually motivated by the effort 
of each player with an intention to maximize its revenue. We assume there is no constraint on the agreement that may accomplish among players. And each network player is considered as an optimizing agent and it expects a reaction from other players to its own action, thus its payoff is determined by other player's actions as well. Since game theory cannot tell us what payoff does each outcome yields, we assign rationale payoff for each player for each configuration which is defined in course of modeling the game. The payoffs are measured in the same units.

\subsection{Game Model between Publisher and Telco CDN}

Since we assume there is no constraint on the agreement that may accomplish among players, both the network players can choose their position freely. If Publisher serves the content with priority then Telco CDN can serve it with priority or without priority. Similarly, if Publisher treats contents without priority, Telco CDN can be agree to serve it with priority or it can deny serving with priority. Definition of priority for Publisher and Telco CDNs are as follows. In Publisher's point of view priority means increasing load. In Telco CDN's point of view, providing priority service means, choosing the best underlying internet paths that one caching server of Telco CDN has to go through to communicate with another Telco CDN's (or Telcos or ISPs) caching server or the customer website and avoid the congested routes. Moreover to ensure priority service (i.e., QoE) Telco CDN will cache the content in its local content routers (within the Telco CDN's network). Besides, it will pursue (by revenue sharing) other peering Telcos or ISPs to do the same.

The Figure 2 depicts the choices and corresponding payoffs for the Publisher and Telco CDN. If Publisher serves the content without priority (normal load), and Telco CDN also treat it as non-priority then both Publisher and Telco CDN have zero payoffs. Caching contents within the Telco CDN (and its peering Telcos \& ISPs) will be on the basis of its popularity index and other conventional strategy. When Publisher serves content without priority (normal load) but Telco CDN treats it with priority, Publisher will have a zero payoff as before. On the other hand Telco CDN will get a negative payoff of -1 . This is an opportunity cost for Telco CDN. It is because Telco CDN could waive the priority (i.e., omit the caching service, stop paying the peering Telcos or ISPs etc.) and use the resource (cache/memory space, monetary transfer to peering Telcos/ISPs etc) for other Publisher's contents. Now, if the Publisher serves the content with priority (increased load) and Telco CDN also treat the content with priority then the Telco CDN will receive a higher payoff 


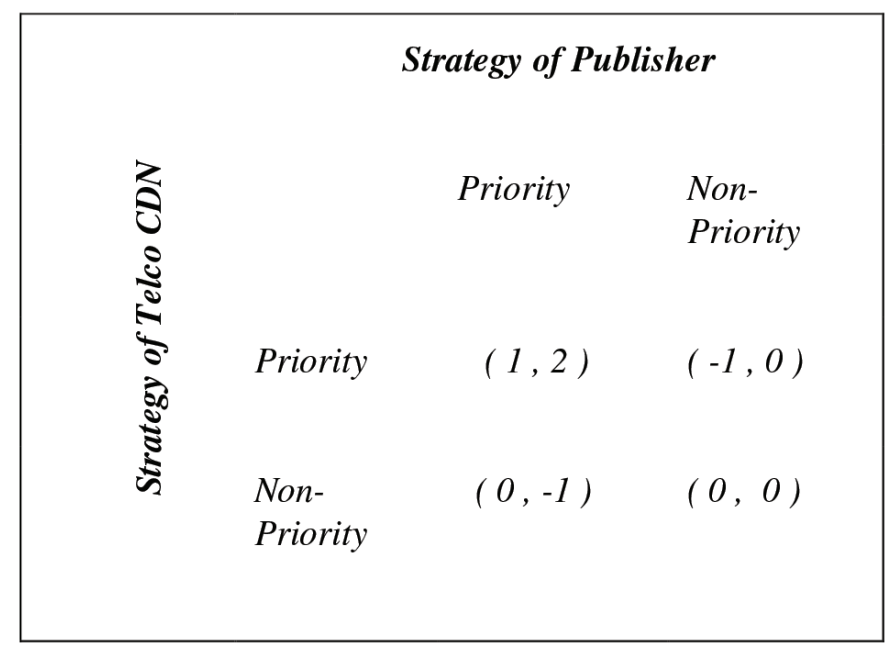

Figure 2 Payoff vectors for Publisher, CDN and TELCO CDN without QoE guarantee

of 2. The reason behind the higher payoff of Telco CDN can be explained in this way. Since the publisher can earn more revenue due to the improved latency of accessing its content, publisher will be happy with the Telco CDN's service hence would possibly renew the contract. The Telco CDN's payoff will be 1 in this case. On the other hand, if the Publisher serves its content with priority (increased load) but Telco CDN treats it as without priority; publisher will lose revenue and (possible lose clients as well; which results a deterioration of the business relationship between the publisher and the Telco CDN. So, in this case the Telco CDN would have a negative payoff of -1 .

\subsection{Revenue Sharing between Telco CDN and Peering Telco CDN (or Telcos or ISPs)}

In this section, we clarify the scenario of revenue sharing between the host Telco CDN (who has agreement with the publisher for content dissemination) and its peer (other Telco CDNs or Telcos or ISPs). The host Telco CDN will earn money from the Publisher and rely on other ISPs or Telcos as a means for last mile connectivity. The peering parties (Telco CDN, Telcos or ISPs) will install extensive cache (Content router) to serve the content to the clients with improved latency hence will provide the quality of experience. Now only charging the end user is not enough for the Telcos (and ISPs) to install and maintain the sufficient caching infrastructure to provide quality 
of experience. As we know the Telco 1.0 business model of charging the enduser for per minute or for per Megabyte is under pressure and new business models for the distribution of content and transportation of data are being developed and appreciated. Operators will need to be capable of charging different network players including end-users, service providers, third-parties (example advertisers) on a real-time basis for provision of guaranteed quality of service (QoS) [18]. So, according to our proposal the peering parties (Telco CDN, Telcos or ISPs) will charge the host Telco CDN for providing the caching service for the content that it access from that host Telco CDN. And the Smart pipes will be used to Charge appropriately for use of the network $[17,18]$.

\section{Three Player Game Model for ICN}

In this section, we provide two separate game models for the three network players; Publisher, Global CDN and Telcos (or ISPs). This game model is also based on the content distribution assumptions described in Section 3 and it holds the other necessary assumptions mentioned in Section 4 as well. In Subsection 6.1, we present the game where there is no agreement for providing Quality of Experience (QoE) among the network players of ICN. We termed this game as a Basic game model among the players. In Subsection 6.2, we consider the Quality of Experience (QoE) guarantee is an integrated part of the agreement among the network players and accordingly we formulate the game model. In Subsection 6.3, we analyze the business relationship among the network players in case of Quality of Experience provision.

\subsection{Basic Game Model between Publisher, Global CDN and Telcos (or ISPs)}

The Figure 3 shows the choices and corresponding payoffs for the publisher, Global CDN and Telcos (or ISPs). All the network players can choose their position freely. Publisher can serve its content with priority requirements (increased load) or non-priority (with normal load). The case of increasing load by the publisher can be defined as priority for the publisher's point of view. And when the publisher uses the normal load it is termed as non-priority from publisher's perspective as we defined in Section 5.

If publisher increase the load (priority), Global CDN can treat its content with priority or Global CDN can deny giving priority treatment to the its content. Similarly, if Global CDN serves the content with priority, Telcos or ISPs can treat it with priority or without priority. When publisher's content is 


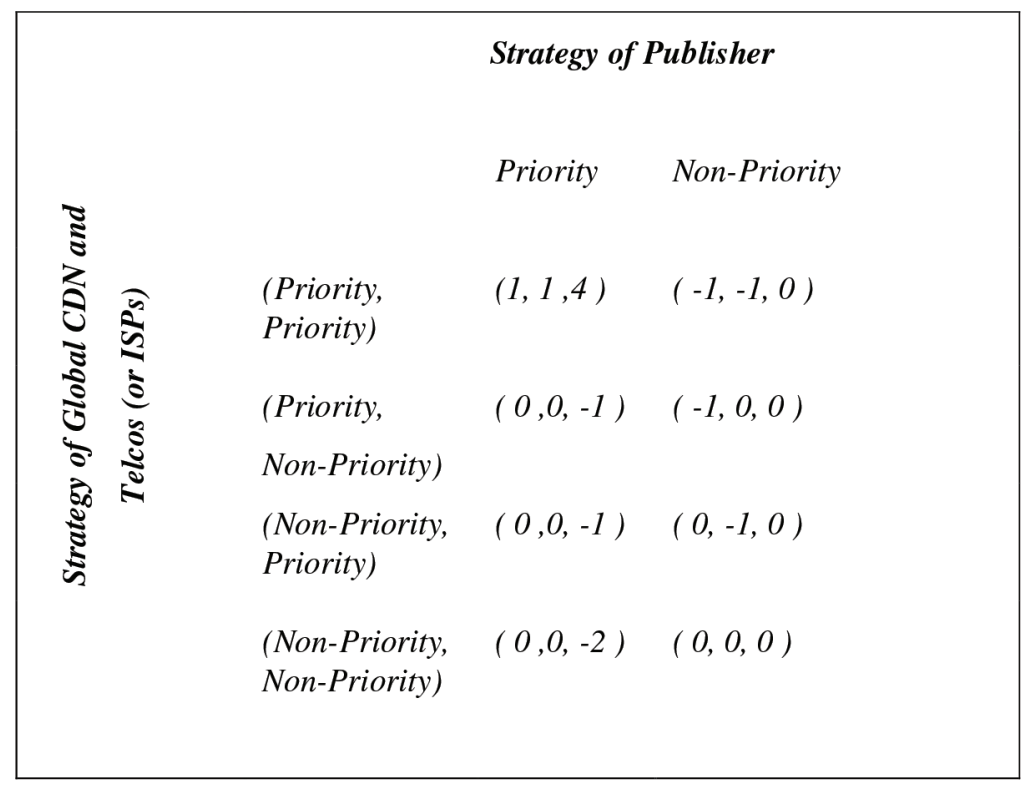

Figure 3 Payoff vectors for Publisher, Global CDN and Telcos or ISPs without QoE guarantee

with normal load (without priority); if Global CDN and Telcos (or ISPs) also treat it as non-priority then all three network players will have zero payoffs. If the Global CDN serve the content with priority and the content is assigned as normal or non-priority by the publisher; Global CDN will get a negative payoff of -1 . This is an opportunity cost for Global CDN. It is because Global CDN could waive the priority and use for other content of the same publisher or other publisher which deserves priority service. If Telcos (or ISPs) serves the same content with priority then it will also have a negative payoff of -1 , for the similar reason that Global CDN earns a negative payoff. If the publisher increases the load (the case of priority) and if Global CDN and Telcos (or ISPs) also serve the content with priority then the publisher will receive a high payoff of 4 . The publisher can earn more revenue, and would possibly renew the agreement with the Global CDN; similarly Global CDN will be happy with the Telcos (or ISPs). So, the Global CDN and Telcos (or ISPs) will achieve a payoff of 1 . On the other hand, if the publisher marks its content as priority but Global CDN and Telcos (or ISPs) do not give priority service to the content; the client will not be happy with the service and publisher will end up with loosing revenue and loosing clients. So, in this case the publisher would have negative payoff. 


\subsection{Game Model between Publisher, Global CDN and Telcos (or ISPs) with Quality of Experience (QoE) Choice}

If we take a short look at the kinds of contract that prevail between Global CDN and publisher; we find that from its legacy Global CDN hardly guarantee the publisher about how long it will take for a client to download contents or access contents. Usually, similar to many other internet services, Global CDNs used to sign the fixed fee agreements with no precise service guarantee. However, it changes over time and the contracts include service guarantee like percentage of "up time" or percentage of request that must be served within a specific time stamp. In case of ICN architecture where the service time of a request will be noticeably reduced, it can be easily foreseen that the Quality of Experience guarantee will be an integrated part of the contract. Note that, Global CDN has control over its caching servers and the way they are connected among each other and connected to the business sites. However, Global CDN does not have any control over the caching strategies (on-path or off-path) of the Telcos and ISPs.

We use the term Quality of Experience (QoE) in case of the Telcos and ISPs and Quality of Service (QoS) in case of Global CDN. Since the Quality of Experience (QoE) is used for evaluating the user experience and it is a measure of the customer satisfaction level, we relate it with the deal of Telcos and ISPs. On the other hand since Quality of Service (QoS) cannot evaluate the quality as seen by the end user and its performance indicator is more networkcentric rather than user centric we relate QoS with the deal of Global CDN. Though the term Quality of Experience deserves more holistic evaluation; for the simplicity in the context of our proposed game theory, we define it as follows. When Global CDN give priority to particular publisher's content, it means it is providing the Quality of Service (QoS) for the content. And when Local Telcos and ISPs sufficiently caches the content to realize the optimized delivery of contents to fulfill the user satisfaction, it is providing the QoE for its client for that particular publisher's content which it receive from Global CDN.

Now let us see, how the choice of Quality of Experience (QoE) guarantee works for the three network players. Global CDN will give a guarantee for attaining quality of experience to the clients of the publisher's content, if publisher pay the fee (negotiated) to Global CDN. Global CDN will do the agreement with the publisher on behalf of Telcos and ISPs as well. It is because; publisher will try to minimize the transaction costs to set up business relation with Telcos and ISPs. The cost of publisher would be 2 and adds directly to 


\section{M. Arifuzzaman et al.}

Global CDN's payoffs. And similarly, Global CDN makes QoE agreement with Telcos and ISPs which cost Global CDN 1 and adds directly to Telcos and ISP's payoff. Figure 4 shows the new payoff.

The agreement of the QoE normally works as follows. If Global CDN meets the QoS (and also Telcos/ISPs meet the QoE) then Global CDN will receive an additional payoff of 2 as bonus. The publisher serves its customers well, makes an additional profit of 4 because of it. Its net profit hence will be 2 after paying the bonus to Global CDN. If the QoS doesn't meet then the Global CDN will have to refund the QoE fee plus pay a penalty of 1 to publisher. And the Telcos (or ISPs) also get a bonus of 1 from Global CDN for meeting the QoE and refund the agreement fee plus penalty of 1 in case of failure to meet QoE. Figure 5 depicts the detailed scenario.

\subsection{Analysis of the QoE Choice for Publisher, Global CDN and Telco CDN}

First of all, we are interested to see if the game with QoE choice reaches a stable situation, where none of the network player is interested to change its strategy provided that all other players keep their strategy unchanged. This situation is called Nash Equilibrium. Note that the game can have more than one Nash Equilibriums. By analyzing Figure 5, we see that, by choosing

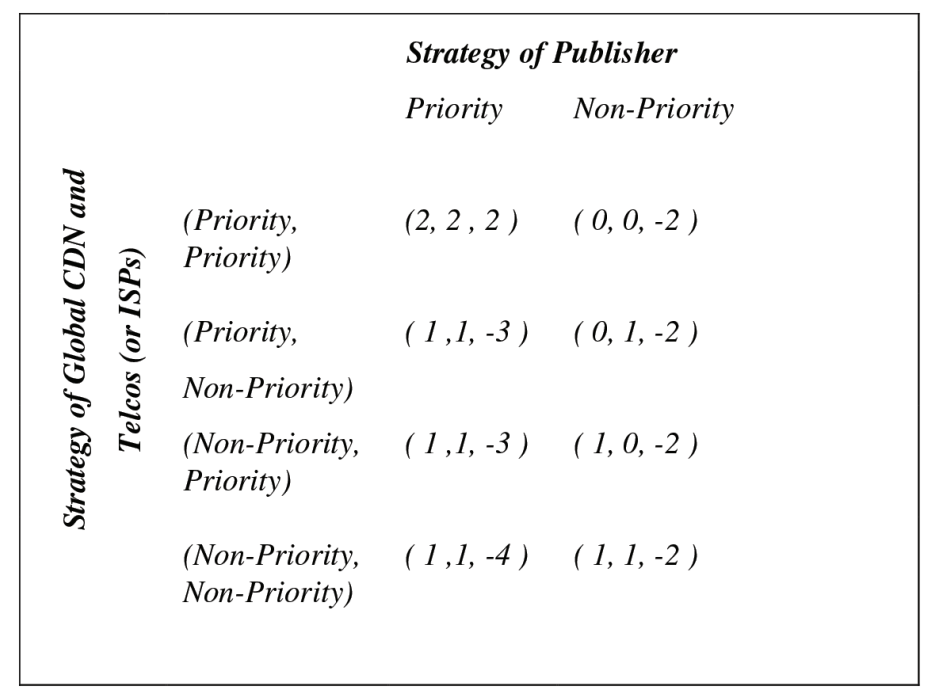

Figure 4 Payoff vectors for Publisher, Global CDN and Telscos or ISPs for making QoE guarantee agreement 


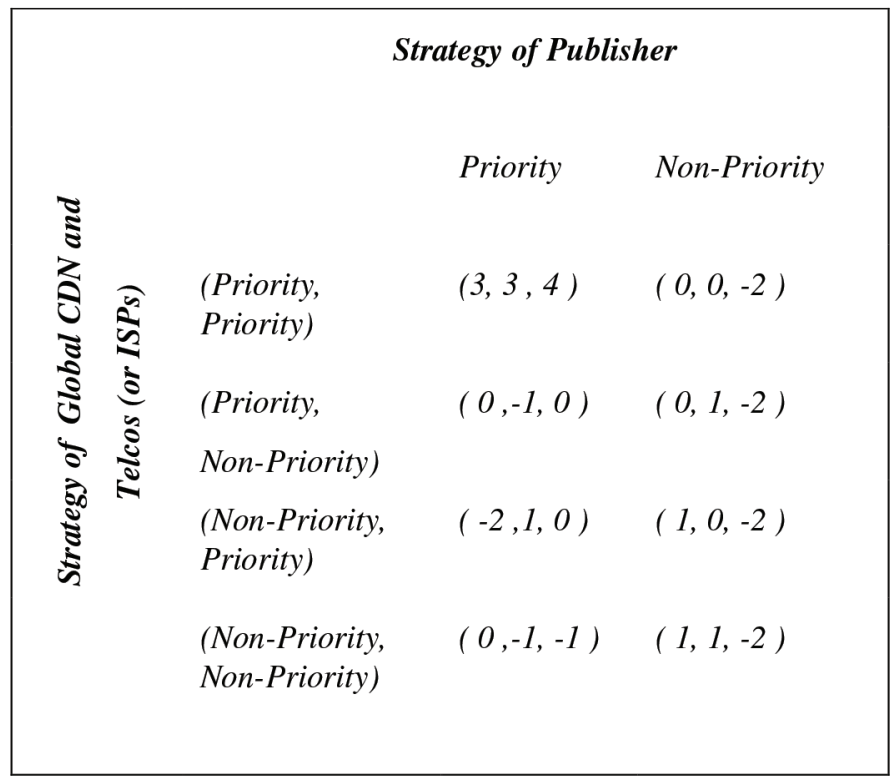

Figure 5 Payoff vectors for Publisher, Global CDN and Tecos or ISPs for guaranteed QoE

the priority option the publisher's payoff becomes $(4,0,0,-1)$ and without giving priority payoff vector is $(-2,-2,-2,-2)$. Similarly, in case of Global $\mathrm{CDN}$, the payoff vector for priority choice is $(3,0,0,0)$ and payoff vector without priority is $(-2,0,1,1$,$) . And Telcos or ISP's payoff vector by choosing$ priority is $(3,1,0,0)$ and payoff vector which it can earn by denying priority $(-1,-1,1,1)$. Now if we consider a particular configuration for example all player choose priority, then the payoff for Publisher, Global CDN and Telcos (or ISPs) becomes $(4,3,3)$ which is the highest payoff for all. So, in this configuration none of the players will change its strategy. Thus we conclude that there will be at least one Nash Equilibrium in the game that we modeled for the quality of service option.

Now, we explain how the QoE choice is the win-win outcomes for all the three network players. We find the solution by using forward deduction scheme. And the comparison will be between the matrices of Figure 3 and Figure 5. First we can remove the column titled non-priority for publisher from the Figure 5. Because, it could have stay with no quality of experience and receive a payoff of 0 instead of -2 . Now publisher will choose priority (payoff 4), rather than choosing general (payoff 0). Now publisher is left with two payoff vector. One is go for quality of service guarantee and earn the 
payoff of $(4,0,0,-1)$ and other is go for $(4,-1,-1,-2)$. Obviously, publisher will go for the first one. Because, it will give it more insured service. Similarly, the quality of service agreement will results Global CDN and Telcos (or ISPs) with a payoff 3 which is higher than the payoff 1 which they receive without going to QoE agreement. Thus the QoE agreement is a win-win situation for all three network player.

\section{Live Streaming Media Broadcast in ICN Perspective}

Achieving high quality and high volume web casts is very challenging. The amount of simultaneous request over such webcast is typically extremely high (for example the expected number of viewers for Tokyo 2020 Olympic). Currently, CDN uses multicasting which is a proven solution. In ICN structure this multicasting can be realized more efficiently. However, the interest of this article is on the economic part of the multicasting for ICN. In multicasting, many request come from the same paths. So, it is more economical to serve the requester by sending the streaming files one time along the path and get as many users as possible to receive the file. Within the extensive cache structure of ICN, the users expressing the interest for the same streaming files can be grouped and sub-grouped optimally which lead to find Steiner minimal multicast tree resulting optimum bandwidth consumption. Hence, by exploiting interest aggregation mechanism and extensive cache structure, there is a great potential for ICN architecture for offering efficient live streaming media broadcasting service. Now, we will show how the CDN and ISP can make their pricing decision about broadcasting the live streaming media over the internet. We show contribution margin for each client subscribing the live stream web cast in the ICN architecture.

First, we consider the lowest three paths of the Figure 6.

For Cache $x_{1}$, combined client value of cache $x_{1}$,

$\mathrm{V}\left(\right.$ Cache $\left.x_{1}\right)=\left(x_{1}+x_{2}+x_{3}\right)$ and the cost $\mathrm{C}\left(\right.$ Cache $\left.x_{1}\right)$ is

$n_{1}$. If $\mathrm{V}\left(\right.$ Cache $\left.x_{1}\right)>\mathrm{C}\left(\right.$ Cache $\left.x_{1}\right)$ then the path from

Cache $x$ to Cache $x_{1}$ will be accepted. Similarly, the Cache $x$ has the combined client value of $\mathrm{V}($ Cache $x)$ will be

$$
\left\{\left(x_{1}+x_{2}+x_{3}\right)+\left(x_{1}+x_{2}\right)+\left(x_{1}+x_{2}+\cdots+x_{n}\right)\right\}
$$




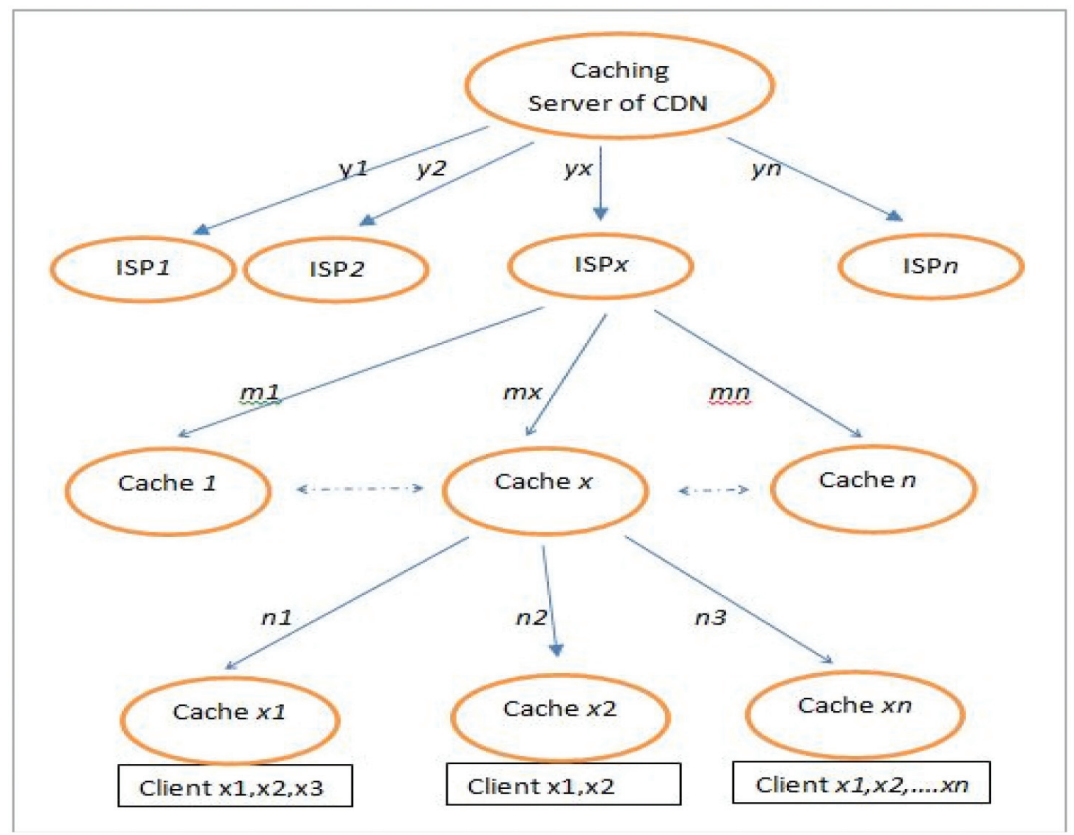

Figure 6 Multicasting decision tree for the real time media streaming for CDNs and ISPs

And the cost is $\mathrm{C}($ Cache $x)=\left(n_{1}+n_{2}+n_{3}\right)$

Now, pie created at cache $x$ can be calculated as value-cost i.e., Pie (Cache $x)=\mathrm{V}($ Cache $x)-\mathrm{C}($ Cache $x)$. Now, the cost of path towards Cache $x$ is $m_{2}$. So, the path, $m_{2}$ will be accepted by ISP $x$ if $m_{2}<$ Pie (Cache $x$ ). Thus, ISP $x$ can calculate its accumulated pie value to make decision whether it is feasible to make contract with CDN. Similarly, CDN will make agree to cast the live stream by comparing the cost and pie value it can accumulate from its different path towards ISPs. We can calculate the contribution margin for each client as well.

Marginal contribution $($ Client $i)=\operatorname{Pie}(\operatorname{Root}(\operatorname{Client} i))-$ Pie $($ Root (Client $i) \backslash$ Client $i$ ).

For example, let us calculate marginal contribution of Client $x_{1}$ (connected to Cache $x_{1}$ ). First if Client $x_{1}$ is not present, we have to see whether the path $n_{1}$ will be accepted by the Cache $x$ or not. If $x_{2}+x_{3}<n_{1}$ then the path will be rejected i,e, not served. In that case, accumulated pie will be

$$
\left\{\left(x_{1}+x_{2}\right)+\left(x_{1}+x_{2}+\cdots+x_{n}\right)-\left(n_{2}+n_{3}\right)\right\}
$$


Hence, the marginal contribution of client, $x_{1}$ is

$$
\begin{aligned}
& \left\{\left(x_{1}+x_{2}+x_{3}\right)+\left(x_{1}+x_{2}\right)+\left(x_{1}+x_{2}+\cdots+x_{n}\right)-\left(n_{1}+n_{2}+n_{3}\right)\right\}-\left\{\left(x_{1}+x_{2}\right)+\right. \\
& \left.\left(x_{1}+x_{2}+\cdots+x_{n}\right)-\left(n_{2}+n_{3}\right)\right\} \\
& \left.=\left\{\left(x_{1}+x_{2}+x_{3}\right)-n_{1}\right)\right\}
\end{aligned}
$$

With this simple mathematical analysis we have shown that the network player like CDNs and the ISPs can find the fair pricing for Live Streaming Media broadcast in ICN.

\section{Conclusion and Future Work}

Information centric network will offer a novel, enhanced and enrich user's experience for accessing content due to improved latency, which results with increased publisher's revenue. With game theoretical model, we have shown how the major network players like Global CDN, Telco CDN, Telcos and ISPs can have a fair share of this increased publisher's revenues. Moreover we address the issue of the guarantee of the Quality of Experience (QoE). With an analysis we have shown that our proposed game reach to Nash Equilibriums and the agreement for Quality of Experience (QoE) choice is a win-win situation for all network players; Publisher, Global CDN and Telco CDN, Telcos and ISPs.

\section{Acknowledgment}

This research was supported by a grant-in-aid from the High-Tech Research Center Project of the Ministry of Education, Culture, Sports, Science and Technology (MEXT), Japan.

\section{References}

[1] D. Trossen, and G.K. Alexandros. "Techno-economic aspects of information-centric networking." Journal of Information Policy 2 (2012).

[2] G. Xylomenos, X. Vasilakos, C. Tsilopoulos, V. A Siris, and G. C. Polyzos, (2012). Caching and mobility support in a publishsubscribe internet architecture. Communications Magazine, IEEE, 50(7), 52-58. 
[3] V. Jacobson, M. Mosko, D. Smetters, and J. J. Garcia-Luna-Aceves, "Content-centric networking: Whitepaper describing future assurable global networks." Response to DARPA RFI SN07-12, 2007.

[4] D. Trossen (ed.), "Architecture definition, component descriptions, and requirements." PSIRP project, 2009.

[5] D. Trossen, M. Sarela, and K. Sollins, "Arguments for an Information Centric Internetworking Architecture." ACM SIGCOMM Comp. Commun. Review, vol 40. No. 2, Apr 2010 pp 27-33.

[6] P. Kwadwo Agyapong, M. Sirbu, "Economic Incentives in InformationCentric Networking: Implications for protocol Design and Public policy." IEEE Communication Magazine December, 2012.

[7] J. Rajahalme, M. Sarela, P. Nikander, S. Tarkoma, "Incentive-Compatible caching and Peering in Data-Oriented Networks", Proc. 2008 ACM CoNEXT Conf. Dec 2008, pp. 62:1-62:-6.

[8] D. Niyato, A.V. Vasilakos, K. Zhu, "Resource and Revenue Sharing with Coalition Formation of Cloud Providers: Game Theoretic Approach." IEEE/ACM International Symposium on Cluster, Cloud and Grid Computing, 2011.

[9] Ng, Chaki, "Game Theory Applications for Content Delivery Networks."

[10] Marco Nicosia, "Internet Video: New Revenue Opportunity for Telecommunications and Cable Providers", Cisco Internet Business Solutions Group (IBSG), July 2010.

[11] Karl, Holger, and Benoit Tremblay. "Document Properties: Document Number: FP7-ICT-2009-5-257448-SAIL/D2. 8." (2012).

[12] B. Chun, K. Chaudhuri, H. Wee, M. Barreno, C. Papadimitriou, and J. Kubiatowicz, "Selfish Caching in Distributed Systems: A Game Theoritic Analysis," PODC, 2004.

[13] P. Golle, K. Leyton-Brown, and I. Mironov, "Incentives for sharing in peer to peer network.", In ACM Conference on Electronic Commerce, 2001.

[14] Arifuzzaman, M.; Keeping Yu, Sato, Takuro, "Content Distribution in Information Centric Network: Economic Incentive Analysis in Game Theoretic Approach", ITU Kaleidoscope conference, Saint Petersburg, Russian Federation, 3-5 June 2014.

[15] T. Koponen, M. Chawla, B. G. Chun, A. Ermolinskiy, K. H. Kim, S. Shenker, I. Stocia, "A Data-oriented (and Beyond) Network Architecture." Proc. SIGCOMM '07, Kyoto, Japan, Aug 27-31, 2007.

[16] B. Ahlgren et al, "Second NetInf Architecture Description." FP7-ICT2007-1-216041-4WARD/D6.2. 
[17] Youngseok Lee, "Telco CDN interconnection for Global Content Exchange", ITU Workshop on "Bridging the Standardization Gap", Vientiane, Lao People's Democratic Republic, 30-31 July 2012.

[18] The value of 'Smart' Pipes to mobile network operators, Chris Barraclough, STL Partners/Telco 2.0, November 2011.

\section{Biographies}

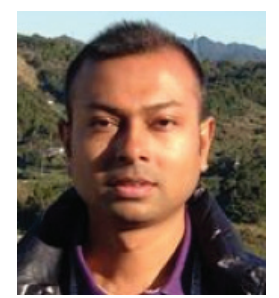

M. Arifuzzaman received the B.Sc. degree in Computer Science \& Engineering from Bangladesh University of Engineering and Technology (BUET) in 2001. He worked as an Assistant professor at IBAIS University, Dhaka, Bangladesh from 2001 to 2005. After that he joined in the Bangladesh Civil Service in 2006 and worked as an Assistant secretary to the Government of the People's Republic of Bangladesh till 2010. He has completed Masters in Global Information and Telecommunication Studies from Waseda University, Tokyo, Japan in 2012. Now he is a PhD candidate at GITS of Waseda University. He received many awards including the best paper award in the ITU Kaleidoscope Conference, Cape Town, South Africa, 12-14 December 2011. His research interests lie in the area of Communication protocols, wireless adhoc and sensor networks, Next Generation Mobile communication systems and Future Internet Architecture. He is a student member of IEEE.

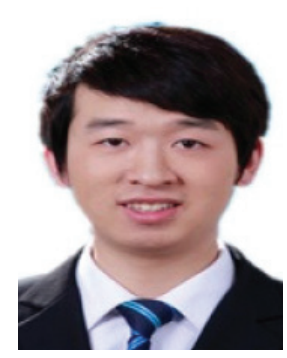

K. Yu was born in China, on January 1988. He received his B.E. and B.Admin. degree from Sichuan Normal University, Sichuan, China in 2010 and University of Electronic Science and Technology of China, Sichuan, China in 2010, 
respectively. He received his M.Sc. degree in Wireless Communication from Waseda University, Tokyo, Japan in 2012. Currently, he is a Ph.D. candidate at Graduate School of Global Information and Telecommunication Studies (GITS), Waseda University, Tokyo, Japan. He is a student member of IEEE. His research interests include smart grid, content-centric networking and their information security.

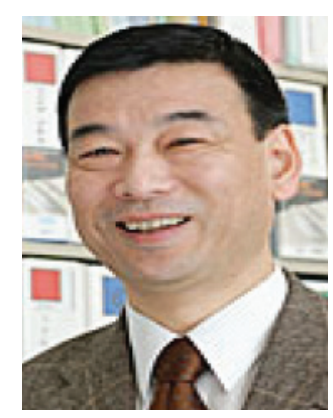

T. Sato received the B.E. and Ph.D. degrees in Electronics Engineering from Niigata University in 1973 and 1993 respectively. He joined the Research and Development Laboratories of OKI Electric Industry Co., Ltd., Tokyo, Japan in 1973 and he has been engaged in research on PCM transmission equipment, mobile communications, data transmission technology and digital signal processing technology. He developed wideband CDMA system for personal communications system and joined the PCS standardization committee in USA and Japan. He contributed in high speed cellular modem standardization for ITU, 2.4GHz PCS standardization for ITA and wireless LAN standardization for IEEE 802.11. He was a Senior Research Manager and Research Director in Communication Systems Laboratory of OKI Electric Industry Co., Ltd. He served as a professor of Niigata Institute of Technology from 1995 and he researched on CDMA, OFDM, personal communication systems and related area. In 2004, he joined as a professor of GITS at Waseda University and currently serving as a Dean of the Graduate School of Global Information and Telecommunication Studies (GITS), Waseda University. His current research interests include Wireless Sensor Network, Mobile IP Network, ICT in Smart Grid, 4G mobile communication systems. He is senior member of IEICE and IEEE. 
